\title{
Low Mortality Rate of Novel Coronavirus Pneumonia (COVID-19) - Outside of the Main Epidemic Area Wuhan
}

\author{
Li Juan Feng ${ }^{1}$, Li Guo², Qing Yang, , , Qing Mei Yang ${ }^{3}$, Yue Yi Zhang ${ }^{1}$, Fei Fei Yang² \\ ${ }^{1}$ Department of Nuclear Medical, The Second Affiliated Hospital of Kunming Medical University, Kunming, P. R. China \\ ${ }^{2}$ Department of Radiology Department Medical, The Second Affiliated Hospital of Kunming Medical University, Kunming, P. R. China \\ ${ }^{3}$ Yuanqinglong Community Health Service Station, Longquan Street, Panlong District, Kunming, P. R. China
}

Email address:

yq7119@hotmail.com (Qing Yang)

${ }^{*}$ Corresponding author

\section{To cite this article:}

Li Juan Feng, Li Guo, Qing Yang, Qing Mei Yang, Yue Yi Zhang, Fei Fei Yang. Low Mortality Rate of Novel Coronavirus Pneumonia (COVID-19) - Outside of the Main Epidemic Area Wuhan. American Journal of Health Research. Vol. 8, No. 3, 2020, pp. 26-31.

doi: $10.11648 /$ j.ajhr.20200803.12

Received: April 7, 2020; Accepted: May 19, 2020; Published: June 4, 2020

\begin{abstract}
Objective: In December 2019, a new coronavirus is spreading in China at a faster pace. This paper focuses on the regional differences in mortality rate to provide useful references and suggestions for the prevention and control of 2019-nCoV pneumonia in other regions of the world. Material/Methods: Data resource: All date of cases In Yunnan Province was confirmed by the author. Another data came from the authority announcement which is update by hours. All data are beginning announced after January 29.2020 when WHO announces a new outbreak of coronavirus pneumonia as a public health emergency of international concern (PHEIC) at 4 a.m. on January 31.2020. Mortality Rate: All statistical analyses were made using SPSS13.0 for Windows software (SPSS Inc., Chicago). The data indicated are expressed as mean \pm standard deviation. The t-test was used to compare the variables between groups. P-value greater than 0.005 was considered as statistically significant, Crude mortality (\%) $=$ number of dead individuals per unit time/number of sicknesses per unit time $\times 1000 \%$. Results: As of 11:34 on April 16, 2020, In China, there were 83,797 confirmed cases, 63 suspected cases, Severe cases 95, 3,352 cases died, 78,504 cases were cured and 713,523 cases were released from medical observation. Hubei has reported 67,803 cases of new crown pneumonia (including 146 cases of clinical diagnosis), among which 50,008 cases (including 146 cases of clinical diagnosis) in Wuhan city, The oversea case is 1,426,071, and only 133818 case died. Crude mortality of Hubei is $47.52(\%)$. The crude mortality rate of Hubei Province is significantly different from other provinces (P-value greater than 0.005). Conclusions: Recent COVID-19 case data show that there are not only more cases but also higher mortality rates in the source areas. It revealed a situation that the lack of medical resources and treatment capacity. Effective epidemic prevention can prevent such a situation. Paying attention to the treatment of light patients is conducive to their conversion into severe, and then reduce the mortality. Low mortality rate of Novel Coronavirus Pneumonia (COVID-19) outside of the main epidemic area Wuhan shows active medical treatment is the key to reduce mortality.
\end{abstract}

Keywords: Novel Coronavirus, 2019-nCoV, COVID-19, Mortality

\section{Introduction}

In December 2019, a new coronavirus achieved cross-species transmission [1, 2], the third successful cross-species transmission in the past 20 years [3]. Compared with the previous two kinds of SARS and MERS, the new coronavirus is not showing weakness [4-6]. It is spreading in China at a faster pace [7].

As of 11:34 on April 16, 2020, In China, there were 83,797 confirmed cases, 63 suspected cases, 3,352 died, 78,504 cases were cured and 713,523 cases were released from medical observation.

In Yunnan Province, where the author lives, 184 confirmed cases were found and no one (only 2 cases) died. From the above data, we found that the mortality of suffer from the 2019-nCoV pneumonia case was significantly different. This paper focuses on the regional differences in mortality rate to provide useful references and suggestions for the prevention 
and control of 2019-nCoV pneumonia in other regions of the world. After all, if the mortality rate is low or zero, then panic is unnecessary. Another influenza also has a certain mortality rate, and what kind of strategy we adopt in the face of 2019-nCoV pneumonia has a wide range of influence which means not only just in health level but also economic and societal levels.

Here I would like to emphasize and draw attention to the fact that the infection of a novel coronavirus does not necessarily lead to viral pneumonia. Quite several patients even have no self-conscious symptoms, but critical cases are always accompanied by pneumonia [8,9]. The mortality of this part of patients is our concern. Reducing mortality is the responsibility and goal of doctors. There is not always bad news outside of the main epidemic area WuHan. 1,426,071 cases were diagnosed and 133,818 cases died outside of China.

\section{Material/Methods}

\subsection{Definition of Disease}

On February 11th, novel coronavirus pneumonia was named COVID-19 by the WHO and the novel coronavirus was named SARS-CoV-2 by the International Committee (ICTV) for classification of viruses.

\subsection{Data Resource}

All date of cases In Yunnan Province was confirmed by the author. Another data came from the authority announcement which is update by hours. All data are beginning announced after January 29.2020 when WHO announces a new outbreak of coronavirus pneumonia as a public health emergency of international concern (PHEIC) at 4 a.m. on January 31.2020.

\subsubsection{Data Source}

All data are from the national health and Health Commission, provincial and municipal health and Health Commission, provincial and municipal governments, Hong Kong, Macao, and Taiwan official channels.

\subsubsection{Data Update Time}

Real-time update of national and provincial urban data, due to the verification of the calculation needs, there will be a certain delay with the official release time. All statistics of epidemic data by 11:34 on April 16, 2020. These data are not the final epidemic data.

\subsubsection{Data Statistics Method}

a) Priority should be given to keeping the national data consistent with the data released by the national health and Health Commission in the morning of each day (at this time, the data of provinces and cities have not been updated in time, and the national data will be greater than the total number of provinces);

b) When the total data published by each province is greater than that published by the state, the national data will be directly switched to the total data of each province; c) National data includes data of Hong Kong, Macao and Taiwan;

The statistics of suspected cases are updated daily, only synchronizing with the total number of the whole country, using the data reported by the national health commission of CHINA every day, and no provincial suspected cases are presented temporarily.

\subsection{Diagnostic Criteria: Recommended by the National Health Commission of China}

\subsubsection{Diagnostic Criteria for Suspected Cases in Provinces Other than Hubei Province}

1. It is consistent with any one of the following epidemiological history and any two clinical manifestations;

2. No clear epidemiological history, in line with 3 of the clinical manifestations.

Epidemiological history

a. Within 14 days before the onset of the disease, there are travel or residence histories of Wuhan city and surrounding areas, or other communities with case reports;

b. there was a history of contact with new coronavirus infected persons (nucleic acid positive) within 14 days before onset.

c. Within 14 days before the onset of the disease, the patients who came from Wuhan city and its surrounding areas, or from the communities with case reports had fever or respiratory symptoms;

d. Clustered disease (refers to the possibility of interpersonal transmission caused by close contact between two or more confirmed cases found in a small area within 14 days, such as a family, a construction site, a unit, a vehicle, etc.).

\section{Clinical manifestation}

a. Fever and / or respiratory symptoms (dry cough, fatigue, etc.);

b. imaging features of new coronavirus pneumonia.

c. In the early stage of the disease, the total number of leukocytes was normal or decreased, or the lymphocyte count was decreased.

Confirmed case

Suspected cases have respiratory tract specimens or blood samples real-time fluorescence RT-PCR detection of new coronavirus nucleic acid positive; virus gene sequencing, and the known new coronavirus highly homologous.

\subsubsection{Diagnostic Criteria for Suspected Cases in Hubei Province}

Criteria for suspected cases in Hubei Province is equivalent to that of non Hubei diagnostic standard, which reduces the imaging features of new coronavirus pneumonia, and it is think that suspected cases with imaging features of new coronavirus pneumonia can be identified as clinically confirmed cases.

\subsubsection{Mortality Rate}

All statistical analyses were made using SPSS13.0 for Windows software (SPSS Inc., Chicago). The data indicated 
are expressed as mean \pm standard deviation. The t-test was used to compare the variables between groups. P-value greater than 0.005 was considered as statistically significant, Crude mortality $(\%)=$ number of dead individuals per unit time/number of sicknesses per unit time $\times 1000 \%$.

\section{Results}

Statistics of national epidemic data by 11:34 on April 16, 2020.

As of 11:34 on April 16, 2020, In China, there were 83,797 confirmed cases, 63 suspected cases, Severe cases 95, 3,352 cases died, 78,504 cases were cured and 713,523 cases were released from medical observation. Hubei has reported 67,803 cases of new crown pneumonia (including 146 cases of clinical diagnosis), among which 50,008 cases (including 146 cases of clinical diagnosis) in Wuhan city, The oversea case is $1,426,071$, and only 133,818 case died. Crude mortality of Hubei is $47.52(\%)$. The crude mortality rate of Hubei Province is significantly different from other provinces (P-value greater than 0.005).

Table 1. All statistics of epidemic data by 11:34 on April 16, 2020.

\begin{tabular}{|c|c|c|c|c|c|}
\hline & Confirmed cases & Suspected cases & Severe cases & Died cases & Crude mortality (\%o) \\
\hline CHINA TOTAL & 83797 & 63 & 95 & 3352 & 40.00 \\
\hline HUBEI & 67803 & 0 & 38 & 3222 & 47.52 \\
\hline YUNNAN & 184 & 0 & 0 & 2 & 10.87 \\
\hline OVERSEA & 1426071 & UNKNOWN & UNKNOWN & 133818 & 93.84 \\
\hline OTHER 33 AREA OF CHINA & 15994 & 63 & 57 & 130 & 8.13 \\
\hline
\end{tabular}

15,994 cases are being reported in the other 33 provinces and over four provinces exceed one thousand cases. 28 province have be confirmed died case reports. (figure 1).

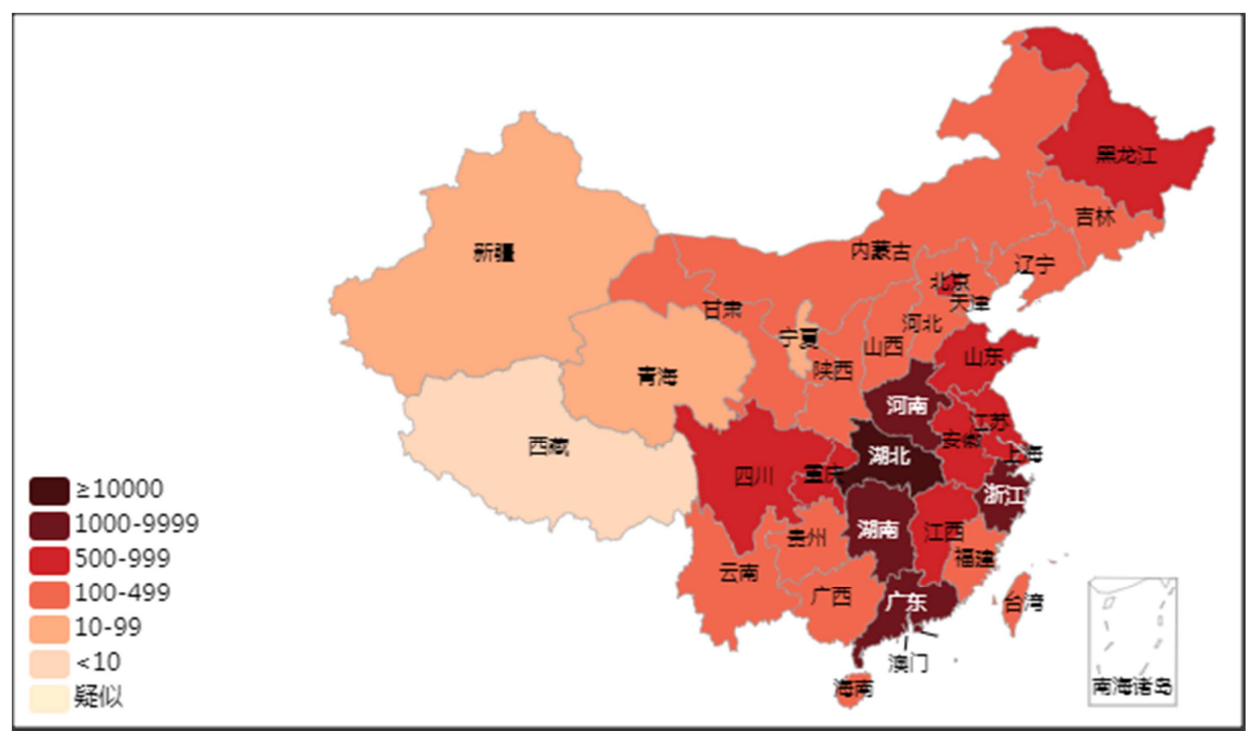

Figure 1. Case distribution map.

Each province died cases reported as table 2 shows. Crude mortality of Hubei is 47.52 (\%o). The crude mortality rate of Hubei Province is significantly different from other provinces (P-value greater than 0.005$)$.

Table 2. Province reported.

\begin{tabular}{llllll}
\hline number & Province name & Confirmed cases & Cured cases & Died cases & Crude mortality (\%) \\
\hline 1 & HUBEI & 67803 & 64435 & 3222 & 47.52 \\
2 & GUANDONG & 1571 & 1471 & 8 & 5.09 \\
3 & HENAI & 1276 & 1254 & 22 & 14.39 \\
4 & ZHEJIANG & 1268 & 1244 & 1 & 0.79 \\
5 & HUNAN & 1019 & 1014 & 4 & 3.93 \\
6 & ANHUI & 991 & 984 & 6 & 1.05 \\
7 & JIANXI & 937 & 936 & 1 & 0.00 \\
8 & JIANSHU & 654 & 642 & 0 & 10.36 \\
9 & CHONGQING & 579 & 570 & 6 & 8.93 \\
10 & SHANDONG & 784 & 761 & 7 & 5.36 \\
11 & SHICHUAN & 560 & 552 & 3 & 15.10 \\
12 & HEILONGJIAN & 861 & 471 & 8 & 13.49 \\
13 & BEIJING & 593 & 503 & & \\
\hline
\end{tabular}




\begin{tabular}{llllll}
\hline number & Province name & Confirmed cases & Cured cases & Died cases & Crude mortality (\%) \\
\hline 14 & SHANGHAI & 628 & 489 & 7 & 11.15 \\
15 & FUJIAN & 353 & 333 & 1 & 2.83 \\
16 & HEIBEI & 328 & 315 & 6 & 18.29 \\
17 & SHANXI & 256 & 251 & 3 & 11.72 \\
18 & GUANXI & 254 & 252 & 2 & 7.87 \\
19 & HAINAN & 168 & 162 & 6 & 35.71 \\
20 & GUIZHOU & 147 & 144 & 2 & 0.00 \\
21 & SHANXI & 194 & 135 & 0 & 13.79 \\
22 & LIAONI & 145 & 138 & 2 & 16.22 \\
23 & TIANJING & 185 & 171 & 14.39 \\
24 & GANSHU & 139 & 136 & 3 & 9.80 \\
25 & JILING & 102 & 96 & 2 & 5.18 \\
26 & NEIMONGU & 193 & 94 & 1 & 39.47 \\
27 & XIJIANG & 76 & 73 & 1 & 0.00 \\
28 & NINXIA & 75 & 75 & 3 & 3.94 \\
29 & HONGAN & 1016 & 459 & 4 & 15.19 \\
30 & TAIWAN & 395 & 155 & 6 & 10.87 \\
31 & YUNNAN & 184 & 176 & 2 & 0.00 \\
32 & QINHAI & 18 & 18 & 0 & 0.00 \\
33 & AOMENG & 45 & 1 & 0 & 0.00 \\
34 & XIZHENG & 1 & 0 & \\
\hline
\end{tabular}

Table 3. HUBEI Province reported.

\begin{tabular}{llllll}
\hline number & AREA name & Confirmed cases & Cured cases & Died cases & Crude mortality (\%) \\
\hline 1 & WUHAN & 50007 & 47283 & 2579 & 51.57 \\
2 & XIAOGAN & 3518 & 3389 & 129 & 36.67 \\
3 & HUANGGANG & 2907 & 2782 & 125 & 43.00 \\
4 & JINGZHOU & 1580 & 1528 & 52 & 32.91 \\
5 & EZHOU & 1394 & 1335 & 59 & 42.32 \\
6 & SUIZHOU & 1307 & 1262 & 45 & 34.43 \\
7 & XIANGYANG & 1175 & 1135 & 40 & 34.04 \\
8 & HUANGSHI & 1015 & 976 & 39 & 44.18 \\
9 & JNGMEN & 928 & 887 & 39.74 \\
10 & YICHANG & 931 & 894 & 37 & 17.94 \\
11 & XIANNING & 836 & 821 & 15 & 11.90 \\
12 & SHIYAN & 672 & 664 & 8 & 38.26 \\
13 & XIANTAO & 575 & 553 & 22 & 30.24 \\
14 & TIANMEN & 496 & 241 & 15 & 27.78 \\
15 & ENSHI & 252 & 189 & 9 & 45.45 \\
16 & QIANJIANG & 198 & 11 & 9 & 0.00 \\
17 & SHENNONGJIA & 11 & & 0 & \\
\hline
\end{tabular}

\section{Discussion}

An important indicator of disease is mortality and the number of infections. There is no doubt that COVID-19 have highly infectious [7, 10]. However, COVID-19 mortality shows its characteristics with the development of disease. In terms of the reported cases, Wuhan has a very high mortality rate, but in other areas, the mortality rate has decreased significantly, and the mortality comparison is statistically significant [11]. The mortality of HUBAEI is $47.52 \%$. The other 33 area of china is $8.13 \%$. The mortality of oversea is 93.84\%o. Although the data will change with time, the characteristics shown in the current data can also play a positive and important role in the prevention and treatment of the disease in the future [12].

So far, six kinds of coronaviruses mainly infect humans, including hcov-229e, hcov-oc43, SARS-CoV, hcov-NL63, hcov-hku1 and MERS-CoV [13]. According to the common degree of the disease, it can be divided into common hcov-229e, hcov-oc43, hcov-NL63, hcov-hku1, and rare SARS-CoV and MERS-CoV [3]. About 30\% of the global respiratory tract infections are caused by four coronaviruses: hcov-229e, hcov-oc43, hcov-NL63, and hcov-hku1 [14]. At the beginning of the disease, they also caused serious symptoms. However, with the emergence of human antibodies, the immune system has adapted to the virus, and now it can only cause mild respiratory symptoms, runny nose, cough, fever, sore throat, etc. Once in a while, pneumonia can be recovered after taking medicine for about one week [15]. I think sars-cov-2 will follow the same rule.

People worry and fear of SARS-COV-2 will affect our strategy of disease control. We must be aware that the control of mortality is directly related to our disease control strategies such as isolation methods and diagnostic criteria [16]. Sometimes the advice of medical experts cannot be taken by policymakers and sometimes policy-making must also consider a series of complex situations, such as religion, customs, medical conditions, etc. I think part of the reason 
why there is a high mortality rate in Wuhan or Hubei Province is because the diagnostic criteria were too strict at the beginning. At the same time, the main reason is the relative shortage of medical resources caused by a large number of cases. Until the patients have serious respiratory disorders and have to be admitted to the hospital for rescue is an actual situation in Wuhan a while ago. This undoubtedly led to an increase in mortality. The low mortality rate outside the epidemic area just explains this situation, where the diagnosed patients get extremely effective medical assistance from the very beginning.

Can we come to such a conclusion? Active and effective medical assistance at the beginning was the cause of low mortality. If we can it will be very important to support the treatment of light patients. The key to reducing the mortality rate is to prevent the patients with mild symptoms turning into severe ones. Of course, it is very important to control the spread of COVID-19 so as not to overload the existing medical treatment on the scale of Wuhan [17]. Now whether the epidemic prevention measures carried out in various parts of China can be effective remains to be confirmed. But all strategies that do not provide actively and acceptable medical assistance must lead to an increase in mortality. We don't lack such a strategy at all.

In CHINA, Some recent strategies are very effective to reduce the mortality rate. For example, the diagnostic standard is no longer the only diagnostic standard of positive nucleic acid test [18], and light patients should be treated appropriately. Two different mortality rates can provide two different models of disease prevention strategies, the Wuhan model and non-Wuhan model. What kind of prevention model to adopt depends on the number of patients and the proportion of medical resources. We should try our best to avoid Wuhan mode.

\section{Conclusions}

Recent COVID-19 case data show that there are not only more cases but also higher mortality rates in the source areas. It revealed a situation that the lack of medical resources and treatment capacity. Effective epidemic prevention can prevent such a situation. Paying attention to the treatment of light patients is conducive to their conversion into severe, and then reduce the mortality. Low mortality rate of Novel Coronavirus Pneumonia (COVID-19) outside of the main epidemic area Wuhan shows active medical treatment is the key to reduce mortality. Thus, If we want to win the battle against coronavirus, we need to avoid the emergence of the Wuhan model as much as possible, especially the early Wuhan model. Once the Wuhan model emerges, it is particularly important to strictly control the spread and actively provide medical assistance. Unfortunately, there is now a shortage of medical resources and a high mortality rate in many regions of the world. For some non-Wuhan model areas, more attention should be paid to the shortage of medical resources, that is, the shortage of doctor resources and materials.

\section{References}

[1] Wan Yushun., Shang Jian., Graham Rachel., Baric Ralph S., Li Fang.(2020). Receptor Recognition by the Novel Coronavirus from Wuhan: an Analysis Based on Decade-Long Structural Studies of SARS Coronavirus. J. Virol., 94 (7), undefined. doi: 10.1128/JVI.00127-20.

[2] Ji Wei., Wang Wei., Zhao Xiaofang., Zai Junjie., Li Xingguang.(2020). Cross-species transmission of the newly identified coronavirus 2019-nCoV. J. Med. Virol., 92 (4), 433-440. doi: 10.1002/jmv.25682.

[3] Guarner Jeannette. (2020). Three Emerging Coronaviruses in Two Decades. Am. J. Clin. Pathol., 153 (4), 420-421. doi: 10.1093/ajcp/aqaa029.

[4] Al-Tawfiq J A., Rodriguez-Morales A J.(2020). Super-sprea ding events and contribution to transmission of MERS, SA RS, and COVID-19. J. Hosp. Infect., undefined (undefined), undefined. doi: 10.1016/j.jhin.2020.04.002.

[5] Wu Joseph T., Leung Kathy., Bushman Mary., Kishore Nis hant., Niehus Rene., de Salazar Pablo M., Cowling Benjam in J., Lipsitch Marc., Leung Gabriel M.(2020). Estimating clinical severity of COVID-19 from the transmission dyna mics in Wuhan, China. Nat. Med., 26 (4), 506-510. doi: 1 0.1038/s41591-020-0822-7.

[6] Mahase Elisabeth.(2020). Coronavirus covid-19 has killed more people than SARS and MERS combined, despite lower case fatality rate. BMJ, 368 (undefined), m641. doi: 10.1136/bmj.m641.

[7] Yan Yuxin., Shin Woo In., Pang Yoong Xin., Meng Yang., Lai Jianchen., You Chong., Zhao Haitao., Lester Edward., Wu Tao., Pang Cheng Heng.(2020). The First 75 Days of Novel Coronavirus (SARS-CoV-2) Outbreak: Recent Advances, Prevention, and Treatment. Int J Environ Res Public Health, 17 (7), undefined. doi: 10.3390/ijerph17072323.

[8] Singhal Tanu.(2020). A Review of Coronavirus Disease-201 9 (COVID-19). Indian J Pediatr, 87 (4), 281-286. doi: 10.1 007/s12098-020-03263-6.

[9] Chu Hin., Chan Jasper Fuk-Woo., Wang Yixin., Yuen Terrence Tsz-Tai., Chai Yue., Hou Yuxin., Shuai Huiping., Yang Dong., Hu Binjie., Huang Xiner., Zhang Xi., Cai Jian-Piao., Zhou Jie., Yuan Shuofeng., Kok Kin-Hang., To Kelvin Kai-Wang., Chan Ivy Hau-Yee., Zhang Anna Jinxia., Sit Ko-Yung., $\mathrm{Au}$ Wing-Kuk., Yuen Kwok-Yung. (2020). Comparative replication and immune activation profiles of SARS-CoV-2 and SARS-CoV in human lungs: an ex vivo study with implications for the pathogenesis of COVID-19. Clin. Infect. Dis., undefined (undefined), undefined. doi: 10.1093/cid/ciaa410.

[10] Chan Jasper Fuk-Woo., Yuan Shuofeng., Kok Kin-Hang., To Kelvin Kai-Wang., Chu Hin., Yang Jin., Xing Fanfan., Liu Jieling., Yip Cyril Chik-Yan., Poon Rosana Wing-Shan., Tsoi Hoi-Wah., Lo Simon Kam-Fai., Chan Kwok-Hung., Poon Vincent Kwok-Man., Chan Wan-Mui., Ip Jonathan Daniel., Cai Jian-Piao., Cheng Vincent Chi-Chung., Chen Honglin., Hui Christopher Kim-Ming., Yuen Kwok-Yung. (2020). A familial cluster of pneumonia associated with the 2019 novel coronavirus indicating person-to-person transmission: a study of a family cluster. Lancet, 395 (10223), 514-523. doi: 10.1016/S0140-6736(20)30154-9. 
[11] Leung Char.(2020). Risk factors for predicting mortality in elderly patients with COVID-19: A review of clinical data in China. Mech. Ageing Dev., 188 (undefined), 111255. doi: 10.1016/j.mad.2020.111255.

[12] Xu Guogang., Yang Yongshi., Du Yingzhen., Peng Fujun., Hu Peng., Wang Runsheng., Yin Ming., Li Tianzhi., Tu Lei. , Sun Jinlyu., Jiang Taijiao., Chang Christopher.(2020). Clin ical Pathway for Early Diagnosis of COVID-19: Updates fr om Experience to Evidence-Based Practice. Clin Rev Aller gy Immunol, undefined (undefined), undefined. doi: 10.100 7/s12016-020-08792-8.

[13] Su Shuo., Wong Gary., Shi Weifeng., Liu Jun., Lai Alexander C K., Zhou Jiyong., Liu Wenjun., Bi Yuhai., Gao George F. (2016). Epidemiology, Genetic Recombination, and Pathogenesis of Coronaviruses. Trends Microbiol., 24 (6), 490-502. doi: 10.1016/j.tim.2016.03.003.

[14] Chen Nanshan., Zhou Min., Dong Xuan., Qu Jieming., Gon g Fengyun., Han Yang., Qiu Yang., Wang Jingli., Liu Ying., Wei Yuan., Xia Jia'an., Yu Ting., Zhang Xinxin., Zhang Li. (2020). Epidemiological and clinical characteristics of $99 \mathrm{c}$ ases of 2019 novel coronavirus pneumonia in Wuhan, Chin a: a descriptive study. Lancet, 395 (10223), 507-513. doi: 1 0.1016/S0140-6736(20)30211-7.

[15] Huang Chaolin., Wang Yeming., Li Xingwang., Ren Lili., Zhao Jianping., Hu Yi., Zhang Li., Fan Guohui., Xu Jiuyang., Gu Xiaoying., Cheng Zhenshun., Yu Ting., Xia Jiaan., Wei Yuan.,
Wu Wenjuan., Xie Xuelei., Yin Wen., Li Hui., Liu Min., Xiao Yan., Gao Hong., Guo Li., Xie Jungang., Wang Guangfa., Jiang Rongmeng., Gao Zhancheng., Jin Qi., Wang Jianwei., Cao Bin. (2020). Clinical features of patients infected with 2019 novel coronavirus in Wuhan, China. Lancet, 395 (10223), 497-506. doi: 10.1016/S0140-6736(20)30183-5.

[16] Dramé Moustapha., Teguo Maturin Tabue., Proye Emeline., Hequet Fanny., Hentzien Maxime., Kanagaratnam Lukshe., Godaert Lidvine.(2020). Should RT-PCR be considered a gold standard in the diagnosis of Covid-19? J. Med. Virol., undefined (undefined), undefined. doi: 10.1002/jmv.25996.

[17] Zhang Chi., Chen Cai., Shen Wei., Tang Feng., Lei Hao., Xie Yu., Cao Zicheng., Tang Kang., Bai Junbo., Xiao Leha n., Xu Yutian., Song Yanxin., Chen Jiwei., Guo Zhihui., G uo Yichen., Wang Xiao., Xu Modi., Zou Huachun., Shu Yu elong., Du Xiangjun.(2020). Impact of population movemen $t$ on the spread of 2019-nCoV in China. Emerg Microbes I nfect, undefined (undefined), 1-28. doi: 10.1080/22221751. 2020.1760143 .

[18] Wang Yun-Yun., Jin Ying-Hui., Ren Xue-Qun., Li Yi-Rong., Zhang Xiao-Chun., Zeng Xian-Tao., Wang Xing-Huan., Zhongnan Hospital of Wuhan University Novel Coronavirus Management and Research Team. (2020). Updating the diagnostic criteria of COVID-19 "suspected case" and "confirmed case" is necessary. Mil Med Res, 7 (1), 17. doi: 10.1186/s40779-020-00245-9. 